\title{
Implementation of a guideline for the treatment of pain, sedation, agitation and neuromuscular blockade in the mechanically ventilated adult patient in the emergency department
}

\author{
This article was published in the following Dove Press journal: \\ Open Access Emergency Medicine \\ 7 April 2011 \\ Number of times this article has been viewed
}

\author{
Kristin E White \\ Paul M Szumital \\ Nicki Gilboy ${ }^{2}$ \\ Hillary A Keenan ${ }^{3}$ \\ Christian Arbelaez ${ }^{2}$ \\ 'Department of Pharmacy Services, \\ ${ }^{2}$ Department of Emergency Medicine, \\ ${ }^{3}$ Center for Clinical Investigation, \\ Brigham and Women's Hospital, \\ Boston, MA, USA
}

\begin{abstract}
Purpose: When emergency department (ED) overcrowding includes admitted mechanically ventilated (MV) critically-ill patients without an open intensive care unit (ICU) bed, emergency providers must deliver ICU level care in the ED. Implementing standardized hospital based clinical guidelines may help providers achieve uniform care standards for assessing and managing pain and sedation for the MV patient.
\end{abstract}

Objective: This paper is a description of a hospital performance improvement project that was implemented in the ED. The objective of this study was to measure the degree of adoption of a hospital-wide clinical guideline for the management of pain, sedation and neuromuscular blockade in MV patients into clinical practice in the ED.

Methods: A retrospective analysis was performed for all mechanically ventilated patients who were admitted from ED to an Intensive Care Unit (ICU). Patient charts were reviewed before (December 2005) and after the implementation of the guideline (June, August, and December 2006). Data was collected and analyzed for the ED visit only and no ICU data was used. The primary outcome was the degree of adoption of the guideline by emergency providers into their daily clinical practice.

Results: A convenience sample of 170 adult MV patients who were admitted to the ICU during the preselected time period was analyzed. There were no demographic differences between groups of patients observed during each month interval, age $(P=0.34)$, gender $(P=0.40)$, race $(P=0.14)$, and Hispanic ethnicity $(P=0.84)$. Overall, there was an increase in the provider use of propofol $(P<0.01)$, RASS sedation scale $(P<0.01)$, and a decrease in the use of a paralytic agent $(P<0.01)$.

Conclusion: There was partial adoption of a guideline into their clinical practice by emergency providers in a busy urban emergency department. Across the 12-month implementation period, there was improvement in the assessment of and use of analgesia and sedation for MV patients.

Keywords: clinical guideline, critical care, ICU, emergency department, sedation, pain, neuromuscular blocker

\section{Purpose}

An increase in the volume and acuity of patients presenting to the emergency department (ED) has led to significant strains on providers. When ED overcrowding includes admitted mechanically ventilated (MV) critically-ill patients without an open intensive care unit (ICU) bed, an added burden of significant importance lies on 
emergency providers, who must deliver ICU level care in the ED. Implementing standardized hospital-based clinical guidelines may help providers achieve uniform care standards for assessing and managing pain and sedation for $\mathrm{MV}$ patients. $^{1-4}$

The importance of providing optimal care for MV adult patients in the ICU has been well documented. The Society of Critical Care Medicine has published practice guidelines for MV patients centered around adequate assessment for and administration of analgesia, sedation, and periodic assessment with a validated tool to determine proper medication effect. ${ }^{5-16}$ Receiving too much or too little medication to manage pain or agitation may lead to adverse outcomes including: delirium; an increase in oxygen consumption; inadvertent removal of devices or catheters; increased risk of infection; and possible post-traumatic stress disorders. ${ }^{11,17-23}$ The emergency medicine literature has focused primarily on rapid sequence intubation and not on the management of the postintubated patient or the use of validated sedation assessment tools for appropriate medication titration to achieve the desired effect for patients while in the ED.

In response to concerns regarding inappropriate analgesia, sedation and use of paralytic agents in MV adult ED patients in our institution, a process improvement team was formed to evaluate the management and find ways to improve care for these patients. A multidisciplinary team was led by an emergency medicine attending physician and included the ED nursing manager, the ED nurse educator, ED staff nurse, an ICU clinical pharmacy specialist, and a pharmacy practice resident.

The process improvement team helped develop an evidence-based clinical guideline for the treatment of pain, agitation and neuromuscular blockade in MV adult patients in the hospital, including the ED and all ICUs (Figure 1). The algorithm addressed the following objectives: 1) providing adequate patient analgesia and reassessment with the Visual Analog Scale (VAS) for titration to effect; 2) providing adequate patient sedation and reassessment with the Richmond Agitation and Sedation Scale (RASS) for titration to effect; and 3) if providing paralysis, after adequate analgesia, sedation, and monitoring with RASS, the ED providers could additionally utilize the Bispectral Index (BIS) to ensure appropriate levels of sedation during paralysis. The objective of this study was to evaluate the degree of adoption of the hospital-wide guideline after implementation into clinical practice in the ED.

\section{Methods \\ Study design}

This study was approved by the hospital investigational review committee. We conducted a retrospective chart review and analysis of all consecutive MV patients admitted to the ICU from the ED during the following four months; baseline (December 2005) and after each phase of implementation (June, August, and December 2006). A report of all patients admitted from the ED to an ICU for each of the four months was generated. A pharmacy practice resident reviewed all admitted patient charts and performed all data collection. An emergency medicine attending physician was available to answer all questions and clarify cases the reviewer had difficulty classifying. All patients who were MV and were at least eighteen years of age were included. No patients were excluded.

Our ED is a level-one trauma and major tertiary referral center with over 56,000 patient visits per year. On average, the hospital admission rate is about $25 \%$ with approximately $40 \mathrm{MV}$ patients admitted to the hospital ICUs per month. Emergency providers were defined as attending physicians, residents, and nurses working in the ED during the study time period. All emergency providers were required to attend a mandatory education and training session which outlined the guideline recommendations for the management of MV patients with adequate analgesia, sedation, and paralysis.

The primary outcome measured was adoption of the guideline into clinical practice by emergency providers. We used the provider order entry (POE) system to obtain the percentage of medications ordered for analgesia, sedation, and paralysis, and the percentage of physician orders for the correct monitoring assessment tools (VAS, RASS, BIS). We used the patient's critical care nursing flow sheet to obtain the percentage of medications that were actually administered for analgesia, sedation, and paralysis and whether or not one of the assessment scales was documented. Data collection occurred from the time the patient entered the ED and received care from the ED providers to the time the patient physically left the ED.

\section{Statistics}

All variables were examined for adherence to the normal distribution visually and by skewness and kurtosis statistics. Chi-square, Fisher's exact test for categorical variables, and the Wilcoxon Rank Sum test for continuous variables were used for determining significant differences between groups. The Cochran-Armitage method was used to test for trends 


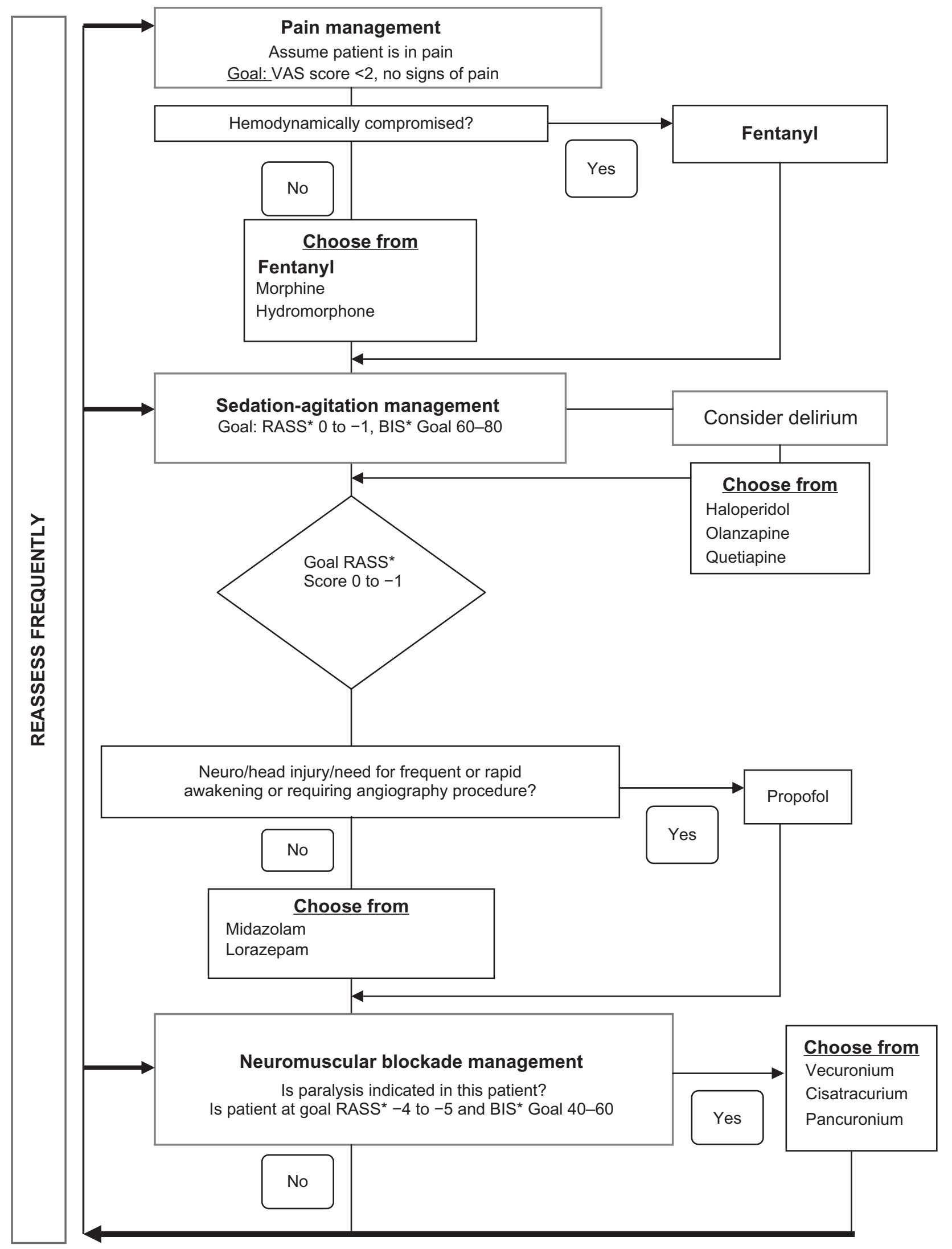

Figure I Clinical algorithm for the hospital-wide guideline for the management of the mechanically vented adult patient. Abbreviations: "VAS, Visual Analog Scale; "RASS, Richmond Agitation and Sedation Scale; "BIS, Bispectral Index. 


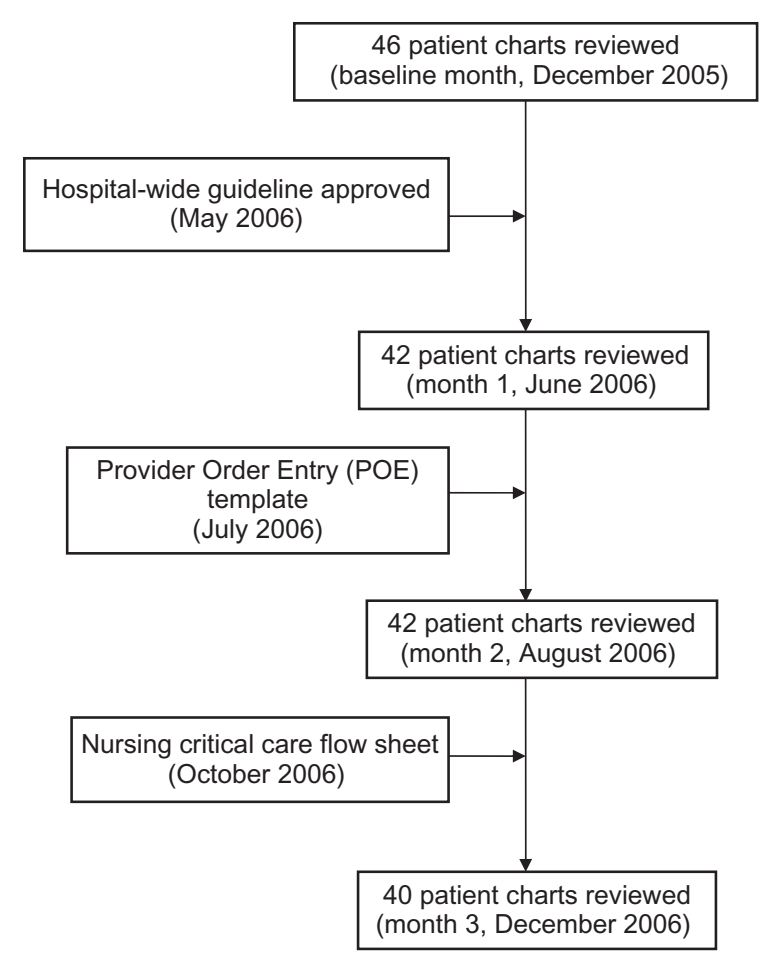

Figure 2 Study cohort after each implementation phase of the hospital-wide guideline for the management of the mechanically vented adult patient in the emergency department (December 2005 and December 2006).

in the distribution of categorical variables across months, whereas, generalized linear regression was used to determine if there was a significant trend across the study period in continuous variables. All analyses were performed using SAS statistical software (v 9.1; SAS Institute, Inc, Cary, NC).

\section{Results}

A total of 170 patient charts were reviewed from the four month intervals ( $\mathrm{n}=46,42,42$, and 40 , respectively). The mean $( \pm \mathrm{SD})$ age of our subjects was $57.9( \pm 19.2), 53 \%$ $(\mathrm{n}=90)$ were female, 68\% $(\mathrm{n}=116)$ were white, and $93 \%(n=157)$ were non-Hispanic. There were no demographic differences between groups of patients observed during each month interval (Table 1$)$, for age $(P=0.34)$, gender $(P=0.40)$, race $(P=0.14)$, or Hispanic ethnicity $(P=0.84)$. The distribution of patients admitted across the different ICUs did not vary across the various months reviewed ( $\%$ admissions: Medical $42 \%$, Surgical 32\%, and Neurological 26\%).

Across the 12-month implementation period, there was a significant increase in the physician ordering of propofol for sedation ( $P$-trend $<0.01 ; 0$ at baseline to $20 \%$ in month 4 ) and nursing documentation of a RASS score in the patient's critical care flow sheet $(P$-trend $<0.01)$. The administration of propofol greatly increased over the study with $33 \%$ of patients receiving the sedative during month 4 versus $0 \%$ in month 1 . The largest increase in provider use of propofol occurred from month $1(0 \%)$ to month $2(24 \%)$ and was most likely related to the hospital approval of propofol continuous infusion for use in MV patients in the ED.

Across all time periods, the percentage of providers documenting the RASS for titration of sedation increased. After the introduction of the patient critical care flow sheet, which included a designated space for RASS documentation, the percentage of patients with a documented score increased by $10 \%$. The documentation of a BIS score in the flow sheet had a trend toward significance $(P$-trend $=0.05)$ across all time points. Emergency providers also ordered and administered less neuromuscular blockade to patients after the introduction of the guideline. Paralytic administration declined over the study period, from $22 \%(\mathrm{n}=10)$ in month 1 to $8 \%(n=3)$ in month $4(P$-trend $=0.06)($ Table 2$)$.

\section{Discussion}

This study demonstrates the partial adoption by emergency providers of a clinical guideline in an urban ED, with an improvement in the use of analgesia, sedation, and paralytics for MV patients. The main goal of the process improvement team was to improve care in the ED by ensuring that all MV patients received the same level of care in the ED and ICUs.

Table I Characteristics of subjects $(n=170)$

\begin{tabular}{|c|c|c|c|c|c|c|c|}
\hline & $\begin{array}{l}\text { All } \\
\%(n)\end{array}$ & $P$-value & $\begin{array}{l}\text { Baseline } \\
n=46\end{array}$ & $\begin{array}{l}\text { Month I } \\
n=42\end{array}$ & $\begin{array}{l}\text { Month } 2 \\
n=42\end{array}$ & $\begin{array}{l}\text { Month } 3 \\
n=40\end{array}$ & P-trend \\
\hline Age (yrs $\pm S D)$ & $57.9 \pm 19.2$ & & $57.9 \pm 22.2$ & $61.4 \pm 18.0$ & $53.8 \pm 19.5$ & $58.4 \pm 16.2$ & \\
\hline Female & $53(90 / 170)$ & 0.47 & 24 & 15 & 21 & 21 & 0.98 \\
\hline White & $68(116 / 170)$ & $<0.01$ & 35 & 24 & 30 & 27 & 0.27 \\
\hline Non-Hispanic & $93(157 / 170)$ & $<0.01$ & 43 & 40 & 37 & 37 & 0.74 \\
\hline Type of ICU & & 0.04 & & & & & 0.60 \\
\hline Medical & $42(7 / / / 70)$ & & 19 & 18 & 18 & 16 & \\
\hline Surgical & $32(55 / 170)$ & & 18 & 12 & 12 & 13 & \\
\hline Neurological & $26(44 / 170)$ & & 9 & 12 & 12 & 11 & \\
\hline
\end{tabular}


Table 2 Adoption of guideline components by emergency providers $(n=170)$

\begin{tabular}{|c|c|c|c|c|c|c|c|}
\hline & $\begin{array}{l}\text { Baseline } \\
n=46 \\
n(\%)\end{array}$ & $\begin{array}{l}\text { Month I } \\
n=42 \\
n(\%)\end{array}$ & $\begin{array}{l}\text { Month } 2 \\
n=42 \\
n(\%)\end{array}$ & $\begin{array}{l}\text { Month } 3 \\
n=40 \\
n(\%)\end{array}$ & $P$-value & $\begin{array}{l}\text { All } \\
n=170 \\
n\end{array}$ & P-trend \\
\hline Analgesia POE Y & $20(43)$ & $23(55)$ & $23(55)$ & $23(58)$ & & 89 & \\
\hline $\mathrm{N}$ & $26(57)$ & $19(45)$ & $19(45)$ & $17(43)$ & 0.60 & 81 & 0.22 \\
\hline Analgesia documented $\mathrm{Y}$ & $23(50)$ & $23(55)$ & $31(74)$ & $25(63)$ & & 102 & \\
\hline $\mathrm{N}$ & $23(50)$ & $19(45)$ & II (26) & $15(38)$ & 0.14 & 68 & 0.14 \\
\hline Sedation POE Y & $20(43)$ & $26(62)$ & $23(55)$ & $20(50)$ & & 89 & \\
\hline $\mathrm{N}$ & $26(57)$ & $16(38)$ & $19(45)$ & $20(50)$ & 0.34 & 81 & 0.59 \\
\hline Sedation documented $\mathrm{Y}$ & $26(57)$ & $27(64)$ & $28(67)$ & $25(63)$ & & 106 & \\
\hline$N$ & $20(43)$ & $15(36)$ & $14(33)$ & $15(38)$ & 0.30 & 64 & 0.58 \\
\hline Propofol POE Y & $0(0)$ & $9(2 I)$ & $9(2 I)$ & $8(20)$ & & 26 & \\
\hline $\mathrm{N}$ & $46(100)$ & $33(79)$ & $33(79)$ & $32(80)$ & $<0.01$ & 144 & $<0.01$ \\
\hline Propofol documented Y & 0 & $10(24)$ & $8(19)$ & $13(33)$ & & 31 & \\
\hline $\mathrm{N}$ & $46(98)$ & $32(76)$ & $34(8 I)$ & $27(67)$ & $<0.01$ & 139 & $<0.01$ \\
\hline Paralytic POE Y & $7(15)$ & $2(5)$ & $3(7)$ & $4(10)$ & & 16 & \\
\hline $\mathrm{N}$ & $39(85)$ & $40(95)$ & $39(93)$ & $36(90)$ & 0.40 & 154 & 0.35 \\
\hline Paralytic documented $Y$ & $10(22)$ & $2(5)$ & $6(14)$ & $3(8)$ & & 21 & \\
\hline$N$ & $36(76)$ & $40(95)$ & $36(86)$ & $37(93)$ & 0.85 & 149 & 0.06 \\
\hline RASS documented $Y$ & $0(0)$ & $0(0)$ & $3(7)$ & $4(10)$ & & 7 & \\
\hline $\mathrm{N}$ & $46(98)$ & $42(100)$ & $39(93)$ & $36(90)$ & 0.04 & 163 & 0.01 \\
\hline BISS documented $Y$ & $0(0)$ & I (24) & $2(5)$ & $3(75)$ & & 6 & \\
\hline $\mathrm{N}$ & $46(98)$ & $41(98)$ & $40(95)$ & $37(93)$ & 0.30 & 164 & 0.05 \\
\hline
\end{tabular}

Abbreviations: N, no; POE, provider order entry; Y, yes.

The most notable finding was the rapid adoption and increased use of the sedative propofol, a medication which had not been previously approved for use in the ED at our institution. We found that most ED providers preferentially used propofol due to its rapid titration ability, despite its designation as a second-line agent for most medical patients in the new algorithm.

We believe that the partial adoption can be attributed to the provider education and training sessions; the new physician order entry template; and the new patient critical care flow sheets that were rolled out clinically with the guideline. For the educational component, the physicians and nurses were given a survey to assess baseline medical knowledge regarding the care of the MV patient. Didactic education and training sessions were then designed and delivered to the emergency providers' needs.

The clinical adjunct tools (physician order entry template and patient critical care flow sheet) were helpful real-time reminders to prompt assessment, documentation and medication titration relative to the VAS, RASS and BIS scales. Despite these tools, changes in clinical practice often take time, resources, and provider buy-in before clinical adoption occurs.

Several trials have assessed the implementation of pain, sedation and delirium monitoring in the ICU; however, to the authors' knowledge this is one of the first attempts at describing and assessing the management of MV patients in the ED. ${ }^{6,15}$
A recent review of the general care of MV patients in the ED has been published emphasizing the importance of extending ICU care standards to MV patients boarding in the ED. This review has further emphasized the need for ED providers to conform to current ICU practice standards to reduce morbidity and mortality of MV ED patients. ${ }^{24}$ The authors believe that the same care goals set by the Society of Critical Care medicine for assessing and treating MV patients' pain and level of sedation may be applied universally to MV ED patients. ${ }^{5}$

Other studies have investigated the implementation of sedation assessment of ICU patients with good adherence. ${ }^{15}$ Compared with a previous guideline implementation study in an ICU setting, our partial adoption of the guideline is less robust, but our observations and challenges faced are similar to what others have described as key components in implementing any protocol in the clinical setting. ${ }^{15}$ These critical elements include having clinical champions and evidence-based guidelines that are clear and easy to use, especially in a large urban setting where there are many rotating patient care providers. ${ }^{6,25-29}$ These clinical champions are either a single person, or more often, a small group of clinicians willing to accept the responsibility to model and encourage a positive change in clinical practice. Clinical pharmacists were not physically present in the ED at the time of our study but may be well positioned to become a part of such efforts in process improvement projects that occur in 
the ED. As ED-based pharmacists increase, there is a clear opportunity for pharmacists to lead these efforts within a multidisciplinary team. Dedicated clinical pharmacists now cover our ED full-time, and are available to give providers real-time feedback in their use of the guideline and management of MV patients. ${ }^{30}$

\section{Limitations}

The results of this study should be interpreted in the context of its limitations. Firstly, this was a single-center retrospective chart review that was not powered a priori for the primary outcomes. It captured adherence with the guideline rather than incidence or ability of achieving the clinical goals as set out by the guideline, as adherence was accepted if there was a single order or recording for each category measured instead of a true assessment of outcomes. We were unable to differentiate between occurrence and patient satisfaction or percentage of time at goal. Patients' satisfaction and comfort was not directly assessed. Some of the documented metrics are suboptimal and may be reflective of new practice changes. Also, our results show more sedation administered by the nurses than what was actually ordered by the physician, which may be attributed to verbal orders. The development and implementation of a hospital-wide guideline require a clinical champion, provider buy-in, adequate resources, education and training sessions, and protocol-based clinical adjuncts. Future projects should focus on a prospective evaluation of clinical outcomes and the impact of ED-based pharmacists in clinical practice guideline adherence.

\section{Conclusion}

As more acutely-ill patients are being cared for and boarded in the ED, the care of the MV patient should be standard across the hospital, including the ED and ICUs. We have developed and implemented an evidence-based algorithm coupled with clinical adjuncts that prompt providers to use it in clinical practice. In our busy urban ED, there was partial adoption by emergency providers of our hospital-wide guideline. Continued efforts are needed to further enhance our guideline adoption. Clinical pharmacists specializing in emergency medicine have a clear opportunity to participate in multidisciplinary efforts in developing, implementing, and monitoring such clinical guidelines.

\section{Disclosure}

Kristin White, Paul Szumita, Nicki Gilboy, Hilary Keenan and Christian Arbelaez have no affiliation with the manufacturers of any of the products mentioned in the paper.

\section{References}

1. Gray A, Gill S, Airey M, Williams R. Descriptive epidemiology of adult critical care transfers from the emergency department. Emerg Med J. 2003;20(3):242-246.

2. Kress JP, Pohlman AS, O'Connor MF, Hall JB. Daily interruption of sedative infusions in critically ill patients undergoing mechanical ventilation. $N$ Engl J Med. 2000;342(20):1471-1477.

3. Wittbrodt ET. Daily interruption of continuous sedation. Pharmacotherapy. 2005;25(5 Pt 2):3S-7S.

4. Chalfin DB, Trzeciak S, Likourezos A, Baumann BM, Dellinger RP, DELAY-ED study group. Impact of delayed transfer of critically ill patients from the emergency department to the intensive care unit. Crit Care Med. 2007;35(6):1477-1483.

5. Jacobi J, Fraser GL, Coursin DB, et al. Clinical practice guidelines for the sustained use of sedatives and analgesics in the critically ill adult. Crit Care Med. 2002;30(1):119-141.

6. Chanques G, Jaber S, Barbotte E, et al. Impact of systematic evaluation of pain and agitation in an intensive care unit. Crit Care Med. 2006; 34(6):1691-1699.

7. Devlin JW, Fraser GL, Kanji S, Riker RR. Sedation assessment in critically ill adults. Ann Pharmacother. 2001;35(12):1624-1632.

8. Ely EW, Gautam S, Margolin R, et al. The impact of delirium in the intensive care unit on hospital length of stay. Intensive Care Med.2001; 27(12):1892-1900

9. Ely EW, Shintani A, Truman B, et al. Delirium as a predictor of mortality in mechanically ventilated patients in the intensive care unit. JAMA. 2004;291(14):1753-1762.

10. McGuire BE, Basten CJ, Ryan CJ, Gallagher J. Intensive care unit syndrome: a dangerous misnomer. Arch Intern Med. 2000;160(7): 906-909.

11. Watson BD, Kane-Gill SL. Sedation assessment in critically ill adults: 2001-2004 update. Ann Pharmacother. 2004;38(11):1898-1906.

12. Venn RM, Bryant A, Hall GM, Grounds RM. Effects of dexmedetomidine on adrenocortical function, and the cardiovascular, endocrine and inflammatory responses in post-operative patients needing sedation in the intensive care unit. Br J Anaesth. 2001 May;86(5):650-656.

13. Riker RR, Fraser GL. Adverse events associated with sedatives, analgesics, and other drugs that provide patient comfort in the intensive care unit. Pharmacotherapy. 2005;25(5 Pt 2):8S-18S.

14. Granberg Axell AI, Malmros CW, Bergbom IL, Lundberg DB. Intensive care unit syndrome/delirium is associated with anemia, drug therapy and duration of ventilation treatment. Acta Anaesthesiol Scand. 2002; 46(6):726-731.

15. Pun BT, Gordon SM, Peterson JF, et al. Large-scale implementation of sedation and delirium monitoring in the intensive care unit: a report from two medical centers. Crit Care Med. 2005;33(6):1199-1205.

16. Arbour R. A continuous quality improvement approach to improving clinical practice in the areas of sedation, analgesia, and neuromuscular blockade. J Contin Educ Nurs. 2003;34(2):64, 71; quiz 90-91.

17. Burton JH, Miner JR, Shipley ER, Strout TD, Becker C, Thode HC Jr. Propofol for emergency department procedural sedation and analgesia: a tale of three centers. Acad Emerg Med. 2006;13(1):24-30.

18. Miner JR, Biros MH, Seigel T, Ross K. The utility of the bispectral index in procedural sedation with propofol in the emergency department. Acad Emerg Med. 2005;12(3):190-196.

19. Sakles JC, Laurin EG, Rantapaa AA, Panacek EA. Airway management in the emergency department: a one-year study of 610 tracheal intubations. Ann Emerg Med. 1998;31(3):325-332.

20. Chao A, Huang CH, Pryor JP, Reilly PM, Schwab CW. Analgesic use in intubated patients during acute resuscitation. J Trauma. 2006;60(3): 579-582.

21. Young C, Knudsen N, Hilton A, Reves JG. Sedation in the intensive care unit. Crit Care Med. 2000;28(3):854-866.

22. Neighbor ML, Honner S, Kohn MA. Factors affecting emergency department opioid administration to severely injured patients. Acad Emerg Med. 2004;11(12):1290-1296. 
23. Adnet F, Minadeo JP, Finot MA, et al. A survey of sedation protocols used for emergency endotracheal intubation in poisoned patients in the French prehospital medical system. Eur J Emerg Med. 1998;5(4): 415-419.

24. Wood S, Winters ME. Care of the Intubated Emergency Department Patient. J Emerg Med. April 2, 2010. [Epub ahead of print].

25. Ricard-Hibon A, Chollet C, Belpomme V, Duchateau FX, Marty J. Epidemiology of adverse effects of prehospital sedation analgesia. Am J Emerg Med. 2003;21(6):461-466.

26. Davis DA, Taylor-Vaisey A. Translating guidelines into practice. A systematic review of theoretic concepts, practical experience and research evidence in the adoption of clinical practice guidelines. CMAJ. 1997;157(4):408-416.
27. Anger KE, Szumita PM. Barriers to glucose control in the intensive care unit. Pharmacotherapy. 2006;26(2):214-228.

28. Rogers EM. Lessons for guidelines from the diffusion of innovations. Jt Comm J Qual Improv. 1995;21(7):324-328.

29. Kahn JM, Goss CH, Heagerty PJ, Kramer AA, O’Brien CR, Rubenfeld GD. Hospital volume and the outcomes of mechanical ventilation. $N$ Engl J Med. 2006;355(1):41-50.

30. Marshall J, Finn CA, Theodore AC. Impact of a clinical pharmacistenforced intensive care unit sedation protocol on duration of mechanical ventilation and hospital stay. Crit Care Med. 2008;36(2):427-433.

\section{Publish your work in this journal}

Open Access Emergency Medicine is an international, peer-reviewed, open access journal publishing original research, reports, editorials, reviews and commentaries on all aspects of emergency medicine. The manuscript management system is completely online and includes a very quick and fair peer-review system, which is all easy to use.

\section{Dovepress}

Visit http://www.dovepress.com/testimonials.php to read real quotes from published authors. 